he adds to the $W$ 's linear combinations of the values of $u$ and its first $n-1$ derivatives at any finite number of points interior to the interval. The Green's function for this system is then defined by the same formula as is used by Birkhoff and it is found that the above integral converges to $f(x)$ provided $f(x)$ has a certain number of derivatives, which number never need exceed $n$, and provided certain determinants formed from the constants of the auxiliary conditions do not vanish. In the case $n$ is even it is further necessary to assume that the second point from either end of the interval is farther from that end than the first point from the other end is from that end.

24. Professor Huntington's paper refers to the theorem of Duhamel already discussed by Osgood, R. L. Moore, and Bliss in the Annals of Mathematics for 1903, 1912, and 1914, namely: If $\alpha_{1}, \alpha_{2}, \cdots, \alpha_{n}$ and $\beta_{1}, \beta_{2}, \cdots, \beta_{n}$ are two sets of infinitesimals such that $\lim _{n=\infty}\left(\beta_{i} / \alpha_{i}\right)=1$; and if $\lim \left[\alpha_{1}+\alpha_{2}+\cdots+\alpha_{n}\right]=a$ exists, then $\lim _{n=\infty}\left[\beta_{1}+\beta_{2}+\cdots+\beta_{n}\right]$ will also exist, and be equal to $a$. The following example of the failure of this theorem is simpler than examples that have been previously given: Let $\alpha_{i}=a / n$, and $\beta_{i}=a / n+2 i c / n^{2}$, where $a$ and $c$ are fixed constants. Then $\lim \beta_{i} / \alpha_{i}=1$ as $n=\infty$; but $\lim \Sigma \alpha_{i}=a$, while $\lim \Sigma \beta_{i}=a+c$.

25. Professor Osgood's paper appeared in full in the June Bulletin.

26. Professor Wilson's paper appeared in full in the May Bulletin.

$$
\begin{gathered}
\text { F. N. Cole, } \\
\text { Secretary. }
\end{gathered}
$$

\title{
APPLICATION OF AN EQUATION IN VARIABLE DIFFERENCES TO INTEGRAL EQUATIONS.
}

BY PROFESSOR G. C. EVANS.

(Read before the American Mathematical Society, April 29, 1916.)

IT is known that if the kernel of an integral equation of Volterra type is in the simple form of the difference alone of the 
two variables, then the kernel of the resolvent equation is in the same form. We shall see presently that this result also holds for the kernel of the Fredholm equation, provided that it is periodic, the period being equal to the interval of integration. Moreover, the same condition of periodicity is sufficient to make the kernel of the resolvent equation of the same form as the original kernel when the latter is expressed by the formula

$$
K(x, y)=\Psi(x+y)+\theta(x-y) .
$$

An obvious way to approach the problem is to make use of the partial differential equations

$$
\frac{\partial K}{\partial x}+\frac{\partial K}{\partial y}=0 \text { and } \frac{\partial^{2} K}{\partial x^{2}}=\frac{\partial^{2} K}{\partial y^{2}},
$$

which say respectively that the kernel $K(x, y)$ is a function of the difference of the two variables, or a function of the form (1). The objection to this method is that there is nothing in the theory of the Fredholm equation which demands the existence of derivatives, and nothing which directly refers to them in the statement of the problem.

Less immediate, and also less restrictive, is a treatment by means of Fourier series. The coefficients in the trigonometric development of the resolvent kernel have simple expressions in terms of those of the given kernel, on account of the special form of the latter. One can, in fact, get an instructive aperçu of the facts in the general problem of the Fredholm equation by considering, with these elementary methods, this special case. Here again, however, more seems to be demanded in the nature of the kernel than should be called for by the question which is the subject of this paper.

\section{§1. Some Difference Equations in Variable Differences.}

1. A necessary and sufficient condition that a function $F(x, y)$ be in the form $f(x+y)$ is that it admit the substitution $x^{\prime}=x+t, y^{\prime}=y-t$, where $t$ is arbitrary. Likewise, a necessary and sufficient condition that a function be in the form $\varphi(x-y)$ is that it admit the substitution $x^{\prime}=x+t$, $y^{\prime}=y+t$. These conditions may be respectively expressed by means of the difference equations 


$$
\begin{aligned}
& F(x+t, y-t)-F(x, y)=0, \\
& F(x+t, y+t)-F(x, y)=0 .
\end{aligned}
$$

2. In order to distinguish the more interesting case where $K(x, y)$ is of the form (1), let us assume at first that $K(x, y)$ is defined everywhere in the plane except at a point set of measure zero.* A necessary and sufficient condition that we may write $K(x, y)$ in the form (1), except possibly at a set of points of measure zero, is that the condition

$$
\begin{aligned}
& K\left(x+t+t^{\prime}, y+t-t^{\prime}\right)-K(x+t, y+t) \\
& -K\left(x+t^{\prime}, y-t^{\prime}\right)+K(x, y)=0
\end{aligned}
$$

shall hold for all values of $x, y, t, t^{\prime}$, except possibly for a point set of zero measure, one of the four points $(x, y),(x+t, y+t)$, $\left(x+t^{\prime}, y-t^{\prime}\right),\left(x+t+t^{\prime}, y+t-t^{\prime}\right)$ lying in that set.

3. The relation (5) says merely that if we take a rectangle of which the sides are parallel to the lines $x+y=0$ and $x-y=0$, the sum of the values of the function at the extremities of one diagonal is equal to the sum of its values at the extremities of the other, i. e., if 1, 2, 3, 4 are the vertices in cyclic order, we have the equation

$$
K_{1}+K_{3}=K_{2}+K_{4} \text {. }
$$

If we have a second rectangle $3,4,5,6$ which has a side in common with the first, it is seen that in the relations

$$
\begin{aligned}
& K_{1}+K_{3}=K_{2}+K_{4}, \\
& K_{3}+K_{5}=K_{4}+K_{6}, \\
& K_{1}+K_{5}=K_{2}+K_{6},
\end{aligned}
$$

the first two imply the third.

Let us define a null set $L$ as made up of the following onedimensional sets. Let it include all the lines $x+y=$ const. or $x-y=$ const. on which there is a not-null set of points where $K(x, y)$ is not defined, and let $x_{0}, y_{0}$ be a point where

* The discussion of this problem in connection with equation (5), below, as applied to point sets in general, offers an interesting generalization of some aspects of the theory of the hyperbolic differential equation: in particular, direct proofs of some of the existence theorems. (See a paper by the author soon to be published. 
$K(x, y)$ still remains defined. On the line $x+y=x_{0}+y_{0}$ there lie at most a null set of points where $K(x, y)$ is not defined. Include in $L$ the lines $x-y=$ const. which go through these points; and operate similarly on the line $x-y=x_{0}-y_{0}$. The total set formed in this way constitutes $L$.

The theorem of Section 2 will be established if we show that a necessary and sufficient condition that $K(x, y)$ be in the form (1), except in $L$, is that the relation (5) hold unless one of the four points mentioned lie in $L$.

4. That the condition is necessary follows at once by direct substitution. In order to show that it is also sufficient, let us write $K(x, y)$ in the form $\Phi(x+y, x-y)$, and consider (5) in the form

$$
\begin{aligned}
\Phi\left(x+y+2 t, x-y+2 t^{\prime}\right)-\Phi(x+y+2 t, x-y) \\
=\Phi\left(x+y, x-y+2 t^{\prime}\right)-\Phi(x+y, x-y),
\end{aligned}
$$

which tells us that the right-hand member is invariant of the substitution $x^{\prime}=x+t, y^{\prime}=y+t$. We can give $t$ then such a value that $x+y+2 t=x_{0}+y_{0}$, and write

$$
\begin{aligned}
& \Phi\left(x_{0}+y_{0}, x-y+2 t^{\prime}\right)-\Phi\left(x_{0}+y_{0}, x-y\right) \\
& \quad=\Phi\left(x+y, x-y+2 t^{\prime}\right)-\Phi(x+y, x-y) \\
& \begin{array}{r}
\text { or } \quad \begin{array}{r}
\Phi\left(x+y, x-y+2 t^{\prime}\right)-\Phi\left(x_{0}+y_{0}, x-y+2 t^{\prime}\right) \\
=
\end{array}(x+y, x-y)-\Phi\left(x_{0}+y_{0}, x-y\right) .
\end{array}
\end{aligned}
$$

The right-hand member of this equation is thus seen to be invariant of the substitution $x^{\prime}=x+t^{\prime}, y^{\prime}=y-t^{\prime}$, wherever it is defined, and so we can give to $t^{\prime}$ a value such that $x-y+2 t^{\prime}=x_{0}-y_{0}$. Hence we have

$$
\begin{aligned}
\Phi\left(x+y, x_{0}-y_{0}\right)- & \Phi\left(x_{0}+y_{0}, x_{0}-y_{0}\right) \\
& =\Phi(x+y, x-y)-\Phi\left(x_{0}+y_{0}, x-y\right)
\end{aligned}
$$

or

(7) $K(x, y)=\Phi\left(x+y, x_{0}-y_{0}\right)+\Phi\left(x_{0}+y_{0}, x-y\right)$

$$
-\Phi\left(x_{0}+y_{0}, x_{0}-y_{0}\right)
$$

which proves the theorem. This result can also be obtained by a more geometrical treatment. 
5. If instead of being defined over the entire plane, the function $K(x, y)$ is defined over the rectangle $R: a<x<A$, $b<y<B$, it may be extended over the entire plane by first defining it in the interior of the circumscribed rectangle with sides parallel to the lines $x+y=0$ and $x-y=0$, and then defining it as having the value zero on the boundary of this rectangle and over the rest of the plane. The definition in this circumscribed rectangle is established by means of the relation $\left(5^{\prime}\right)$, and its uniqueness follows almost immediately by means of the relation (6). By then applying the theorem of Section 2 we have the following

Corollary 1. The theorem of Section 2 holds, if instead of being defined over the entire plane, except for a point set of measure zero, it is defined, with the same exception, over the rectangle $R: a<x<A, b<y<B$.

We have also the following theorems:

Corollary 2. The condition (5) will still be necessary and sufficient if we add the restriction that $|t|$ and $\left|t^{\prime}\right|$ are to be less than $\eta$, where $\eta$ is assigned arbitrarily, positive, dependent on $x, y$ if desired.

This theorem is deduced immediately with the help of equation (6).

Corollary 3. If the function $K(x, y)$ is continuous at the points in which it is defined, the condition (5) may be replaced by one in which $t^{\prime}=t, i$. e., by the condition

$$
K(x+2 t, y)-K(x+t, y+t)-K(x+t, y-t)
$$

In fact, from (8) it follows that

$$
+K(x, y)=0 .
$$

$$
\begin{aligned}
K(x+m t+n t, y+m t & -n t)-K(x+m t, y+m t) \\
& -K(x+n t, y-n t)+K(x, y)=0,
\end{aligned}
$$

where $m$ and $n$ are integers, and since every point in $R$ is a point of the form $(x+m t+n t, y+m t-n t)$ or a limiting point of such points, we have the relation (5).

COROLLARY 4. The relation (5) may be replaced by the one obtained from it by interchanging $t$ and $t^{\prime}$, or by both relations together, and the theorem will still hold.

CoRollary 5. A necessary and sufficient condition that we may write $K(x, y)$ in the form 


$$
K(x, y)=f(x)+g(y)
$$

is that

$$
\begin{aligned}
K\left(x+t, y+t^{\prime}\right)-K\left(x, y+t^{\prime}\right)-K(x & +t, y) \\
& +K(x, y)=0 .
\end{aligned}
$$

\section{§2. Periodicity.}

6. The kernel of an integral equation and the kernel of the resolvent equation are connected by Volterra's relation. If we are dealing with the equation of Fredholm type containing a parameter $\lambda$, the relation has the well known form

$$
\begin{aligned}
K(x, y)+k_{\lambda}(x, y) & =\lambda \int_{a}^{b} K(x, \xi) k_{\lambda}(\xi, y) d \xi \\
& =\lambda \int_{a}^{b} k_{\lambda}(x, \xi) K(\xi, y) d \xi
\end{aligned}
$$

For generality the integral may be taken in the Lebesgue sense. From (10) it follows obviously that if $K(x, y)$ is periodic in $x$ with a certain period, $k_{\lambda}(x, y)$ is periodic in $x$ with the same period; and similarly for $y$.

In regard to the kernels of the form (1), in which we are more specially interested, a necessary and sufficient condition that $K(x, y)$ be periodic, with period $p$, in both arguments, is that $\Psi(x+y)$ and $\Theta(x-y)$, as functions of a single variable, be each periodic with the same period $p$. Obviously the condition is sufficient. To show that it is necessary, notice that we have

$$
K(x+t, y+t)-K(x, y)=\Psi(x+y+2 t)-\Psi(x+y),
$$

whence, taking $t=p$,

$$
\Psi(x+y+2 p)=\Psi(x+y) .
$$

But also

$$
\begin{aligned}
& \begin{aligned}
K(x+p+t, y+t) & -K(x+p, y) \\
& =\Psi(x+y+p+2 t)-\Psi(x+y+p),
\end{aligned} \\
& \text { so that } \\
& \begin{aligned}
\Psi(x+y+p+2 t)-\Psi(x+y+p) \\
=
\end{aligned} \\
& =\Psi(x+y+2 t)-\Psi(x+y),
\end{aligned}
$$


and if $t=\frac{1}{2} p$,

$$
\Psi(x+y+2 p)+\Psi(x+y)=2 \Psi(x+y+p) .
$$

Comparing this with (11), we have

$$
\Psi(x+y+p)=\Psi(x+y) .
$$

From this and the periodicity of $K(x, y)$ it follows that

$$
\Theta(x-y+p)=\Theta(x-y) .
$$

Nothing is changed in these theorems if we except a point set of zero measure (in the case of the second theorem, a point set of the type $L$ ).

\section{§3. Kernels of the Form $\Psi(x+y)+\Theta(x-y)$.}

7. Let us understand the integrals to be taken in the Lebesgue sense, and the function $K(x, y)$ to be summable, with its square, over any two-dimensional region. The Volterra relations (10) hold with this assumption except at a point set of measure zero, and by giving a complete definition of the function $K(x, y)$ they can be made to define a function $k(x, y)$ at every point, finite or infinite, as the case may be. We have the following theorem:

Theorem 1. If, except at the points of a set $L$ (see Section 3) of measure zero, the kernel $K(x, y)$ can be written in the form (1), in which the functions $\Psi$ and $\Theta$, as functions of a single argument, are periodic with period $b-a$, then with the same exception, the resolvent kernel $k(x, y)$ can be written in the same form, and has the same properties of periodicity.

As has already been noticed in Section 6 , it is the same thing as above to assume that $K(x, y)$ is periodic in each argument with period $b-a$, and the periodicity of $k(x, y)$ follows from it. By virtue of the assumption of periodicity in regard to $K(x, y)$ the point set $L$ is itself periodic.

8. In order to prove the theorem let us perform on the second of the equations (10) the operations indicated in (5), and also the operation obtained from this by interchanging $t$ and $t^{\prime}$. Let us denote the result of performing these operations on a function $f(x, y)$ by $H_{f}\left(t, t^{\prime} \mid x, y\right)$ and $H_{f}\left(t^{\prime}, t \mid x, y\right)$ respectively. In forming the resulting pair of equations we have to perform such reductions as the following: 


$$
\begin{aligned}
\int_{a}^{b} k_{\lambda}\left(x+t+t^{\prime}, \xi\right) \Psi\left(\xi+y+t-t^{\prime}\right) d \xi \\
=\int_{a+t-t^{\prime}}^{b+t-t^{\prime}} k_{\lambda}\left(x+t+t^{\prime}, \xi-t+t^{\prime}\right) \Psi(\xi+y) d \xi
\end{aligned}
$$

which reduces to

$$
\int_{a}^{b} k_{\lambda}\left(x+t+t^{\prime}, \xi-t+t^{\prime}\right) \Psi(\xi+y) d \xi
$$

on account of the periodicity. ${ }^{*}$ Hence we obtain finally the pair of simultaneous integral equations:

$$
\begin{aligned}
H_{K}\left(t, t^{\prime} \mid x, y\right)+H_{k}\left(t, t^{\prime} \mid x, y\right) & \\
= & \lambda\left[\int_{a}^{b} H_{k}\left(t^{\prime}, t \mid x, \xi\right) \Psi(\xi+y) d \xi\right. \\
& \left.+\int_{a}^{b} H_{k}\left(t, t^{\prime} \mid x, \xi\right) \Theta(\xi-y) d \xi\right], \\
H_{K}\left(t^{\prime}, t \mid x, y\right)+H_{k}\left(t^{\prime}, t \mid x, y\right) & \\
= & \lambda\left[\int_{a}^{b} H_{k}\left(t^{\prime}, t \mid x, \xi\right) \Theta(\xi-y) d \xi\right. \\
& \left.+\int_{a}^{b} H_{k}\left(t, t^{\prime} \mid x, \xi\right) \Psi(\xi+y) d \xi\right] .
\end{aligned}
$$

In this system of equations the kernels are the functions $\Psi$ and $\theta$, the known functions are the $-H_{K}$ and the solutions desired are the functions $H_{k}$. It is worth noticing that since the kernels are independent of $t$ and $t^{\prime}$, any of the functions belonging to $K(x, y)$ gives rise to a solution of the homogeneous system of equations corresponding to (12), and vice versa; hence the characteristic values of $\lambda$ in (12) are merely the roots of $K(x, y)$. In fact if we write $t=t^{\prime}$ in (12), the pair of equations reduce to a single equation of which the kernel is $K(x, y)$. The solutions of (12) will then be determined unless $\lambda$ happens to be root of $K(x, y)$.

Since for given values of $t$ and $t^{\prime}$ the known functions vanish except at the points of a set of zero measure (i. e., except when

* In the treatment by means of the partial differential equation, this change of variable corresponds to an integration by parts. 
one of the seven points $(x, y),(x+t, y+t),(x+t, y-t)$, $\left(x+t^{\prime}, y+t^{\prime}\right), \quad\left(x+t^{\prime}, y-t^{\prime}\right),\left(x+t+t^{\prime}, y+t-t^{\prime}\right)$, $\left(x+t+t^{\prime}, y-t+t^{\prime}\right)$ lie in $\left.L\right)$, the solutions will vanish except at a point set of zero measure. Moreover from the formal character of the solution this set will not contain notnull sets of points on any line parallel to the $X$ or $Y$ axes. Hence the right hand members of (12) will vanish identically, and at all points, even at points of $L$, we shall have the result

$$
H_{k}\left(t, t^{\prime} \mid x, y\right)=-H_{K}\left(t, t^{\prime} \mid x, y\right),
$$

which asserts that $H_{k}\left(t, t^{\prime} \mid x, y\right)$ and $H_{k}\left(t^{\prime}, t \mid x, y\right)$ both vanish for all values of $x, y, t, t^{\prime}$ such that none of the seven points before mentioned lie in $L$. Hence, by means of Corollary 4 in Section $5, k(x, y)$ will have the form (1), except for points of $L$; and the theorem is proved.

9. If instead of being defined over the entire plane, the kernel $K(x, y)$ is defined only for values of $x$ and $y$ between $a$ and $b$, the theorem may be extended to cover this case. For if the kernel is in the form (1), it is defined thereby over the interior of the circumscribing rectangle mentioned in Section 5 . In fact, that is the region of variation for $x$ and $y$ determined by the arguments $x+y$ and $x-y$, on which depend the functions $\Psi$ and $\theta$. In order to complete the definition of the function over the entire plane in the manner best to fit with Theorem 1, let us cover the plane by repeating the circumscribing rectangle with the attached values of $K(x, y)$, thus making $K(x, y)$ periodic in $x$ and $y$ separately with period $2(b-a)$. By means of Theorem 1, then, we have the following theorem:

THEOREM 2. If for values of $x$ and $y$ between $a$ and $b$ the kernel $K(x, y)$ can be written in the form (1), in which the functions $\Psi$ and $\Theta$, as functions of a single argument, are periodic with period $b-a$, the hypothesis holding except at a point set of zero measure, of the form $L$ (see Section 3), then with the same exception, the resolvent kernel $k(x, y)$ can be written in the same form, and has the same properties of periodicity.

10. The corresponding theorem is true for functions of the form

$$
K(x, y)=\Theta(x-y) .
$$

In fact, if we make use of the equation 


$$
K(x+t, y+t)-K(x, y)=0,
$$

we can deduce by means of it, in the same way as before we used the relation (5), the following theorem:

Theorem 3. Theorems 1 and 2 hold if instead of considering kernels of the form (1) we consider kernels of the form (14).

The corresponding theorem is not true for kernels which are functions of $x+y$ alone. In regard to functions of the form (14), however, we may go still further, if they are continuous.

Theorem 4. If $K(x, y)$ is in the form (14) and is continuous, a necessary and sufficient condition that $k(x, y)$ should be in the same form is that $\Theta$, as a function of a single argument, be periodic with period $b-a$.

It may be noticed that if the kernel is a function of $x$ alone, or is a function of $y$ alone, the resolvent kernel is of the same form; but if the kernel is of the form (9), that is, a function of $x$ alone plus a function of $y$ alone, the resolvent kernel is not necessarily of the same form. In fact, if $f_{1}(x)$ is the resolvent associated with $f(x)$, and $g_{1}(y)$ is the resolvent associated with $g(y)$, then the resolvent associated with $f(x)+g(y)-\int_{a}^{b} f(\xi) g(\xi) d \xi$ is $f_{1}(x)+g_{1}(y)-f_{1}(x) g_{1}(y)(b-a)$.*

\section{§. Applications.}

11. On account of the fact that kernels of the form (1) need not be continuous, they may often be used as approximations for particular kernels which are not themselves in that form. The resolvent kernel will then again under the hypothesis of Theorem 1 be in the form (1), and the method of development in trigonometric functions of period $b-a$ yield immediate results. Indeed, functions of the form (1) are determined if they are known along a line $x+y=c_{1}$ and a line $x-y=c_{2}$; a remark which simplifies especially the treatment of the integral equation of Volterra type, since it leaves only the single function $\varphi$ to be calculated. The subject of approximation has not been much studied in connection with integral equations.

* See Section 12 below, also Evans, "L'algebra delle funzioni permutabili e non permutabili," Rendiconti del Circolo Matematico di Palermo, vol. 34, p. 7 . 
12. If $K_{1}(x, y)$ and $K_{2}(x, y)$ are functions of the form (1), then it is easily shown by means of (5) that the integral combination

$$
\int_{a}^{b} K_{1}(x, \xi) K_{2}(\xi, y) d \xi
$$

is of the same form, provided that the hypothesis of periodicity (the period being $b-a$ ) holds for the parts $\Psi_{1}, \theta_{1}$ and $\Psi_{2}, \theta_{2}$ of $K_{1}$ and $K_{2}$. This is the integral combination which has been so extensively studied by Volterra.

Now it is known that if we are given two functions $K_{1}$ and $K_{2}$ of $x$ and $y$, and their respective resolvent kernels $k_{1}$ and $k_{2}$, there may be built up out of them by means of the combination (16) a new kernel and its resolvent; in fact, the function

$$
k_{1}(x, y)+k_{2}(x, y)-\int_{a}^{b} k_{1}(x, \xi) k_{2}(\xi, y) d \xi
$$

is resolvent for the function

$$
K_{2}(x, y)+K_{1}(x, y)-\int_{a}^{b} K_{2}(x, \xi) K_{1}(\xi, y) d \xi . *
$$

We have then the theorem:

If we have $K_{1}(x, y)=\Psi_{1}(x+y)+\theta_{1}(x-y)$ and $K_{2}(x, y)$ $=\Psi_{2}(x+y)+\Theta_{2}(x-y)$, where $\Psi_{1}, \Theta_{1}$ and $\Psi_{2}, \Theta_{2}$, as functions of a single argument, are periodic with period $b-a$, and if we denote by $k_{1}(x, y)$ the function resolvent to $K_{1}(x, y)$, and by $k_{2}(x, y)$ the function resolvent to $K_{2}(x, y)$, then the functions given by (17) and (18) are of the same form, have the same properties of periodicity, and are themselves mutually resolvent kernels.

Rice Institute, April, 1916.

\section{OPERATORS IN VECTOR ANALYSIS.}

BY DR. VINCENT C. POOR.

IN a note in the April Bulletin on " Changing surface to volume integrals," Professor E. B. Wilson asks why my paper in the January Bulletin was not made shorter by using the Gibbs-Wilson notation. While the brevity and suggestiveness

* See the footnote to Section 10. For purposes of symmetry and convenience of statemen we have taken $\lambda=1$ and assumed it not to be a root of $K_{1}$ or $K_{2}$. 